\title{
Elementos da masculinidade que vulnerabilizam homens à morbimortalidade pela COVID-19: revisão integrativa
}

\author{
Elements of masculinity that vulnerabilize men to morbimortality by COVID-19: integrative review \\ Elementos de masculinidad que vulnerabilizan a los hombres a la morbimortalidad por COVID-19: revisión integrativa
}

\begin{abstract}
RESUMO
Objetivou-se conhecer os elementos da masculinidade que vulnerabilizam homens a morbimortalidade pela COVID-19. Trata-se de uma revisão integrativa, realizada no mês de maio de 2020, por meio das plataformas Pubcovid-19 e a Biblioteca Virtual em Saúde. Utilizou-se as seguintes estratégias de busca "Covid" and "Men"; "Covid" and "Men" and "Health"; "Covid" and "Gender identity", apresentando 490 documentos e após os critérios de inclusão totalizaram 07 artigos. Os elementos da masculinidade que incitam a vulnerabilidade do homem à morbimortalidade pela COVID-19 dizem respeito ao provimento familiar e consequente exposição ocupacional no trabalho, estilo de vida não saudável, expressos pelo tabagismo e alcoolismo, violação das regras de distanciamento social, negligência de práticas preventivas, que reverberam no sistema imunológico masculino mais debilitado. Entretanto à literatura também desvelou que a não busca masculina pelos serviços de saúde influenciado pela convicção de invulnerabilidade e pelos discursos políticos que minoram a doença também são influenciadoras na elevação da exposição e mortalidade masculina pela COVID-19. O conhecimento dos elementos que favorecem os homens ao adoecimento e consequente morte pela doença, urge que sejam pensadas estratégias de prevenção para esse público.
\end{abstract}

DESCRITORES: COVID-19; Saúde do homem; Indicadores de Morbimortalidade.

\section{ABSTRACT}

El objetivo fue conocer los elementos de masculinidad que hacen a los hombres vulnerables a la morbilidad y mortalidad por COVID-19. Se trata de una revisión integradora, realizada en mayo de 2020, a través de las plataformas Pubcovid-19 y la Biblioteca Virtual en Salud, se utilizaron las siguientes estrategias de búsqueda "Covid" y "Men"; "Covid" y "Hombres" y "Salud"; "Covid" e "Identidad de género", afirman 490 documentos y luego de los criterios de inclusión, totalizaron 07 artículos. Los elementos de masculinidad que incitan a la vulnerabilidad de los hombres a la morbilidad y mortalidad por COVID-19 se refieren a la provisión familiar y la consiguiente exposición ocupacional en el trabajo, estilo de vida poco saludable, expresado por el tabaquismo y el alcoholismo, violación de las reglas de distancia social, descuido de prácticas, medidas preventivas, que reverberan en el sistema inmunológico masculino debilitado. Sin embargo, la literatura también reveló que la falta de búsqueda masculina de servicios de salud influenciada por la convicción de invulnerabilidad y por los discursos políticos que alivian la enfermedad también están influyendo en el aumento de la exposición y mortalidad masculina por COVID-19. El conocimiento de los elementos que favorecen al hombre a la enfermedad y consecuente muerte por la enfermedad, urge que se piense en prevención para este público.

DESCRIPTORS: Coronavirus Infections; Men's Health; Indicators of Morbidity and Mortality

\section{RESUMEN}

The objective was to know the elements of masculinity that make men vulnerable to morbidity and mortality by COVID-19. It is an integrative review, carried out in May 2020, through the Pubcovid-19 platforms and the Virtual Health Library. The following search strategies "Covid" and "Men" were used; "Covid" and "Men" and "Health"; "Covid" and "Gender identity", state 490 documents and after the inclusion criteria, they totaled 07 articles. The elements of masculinity that incite the vulnerability of men to morbidity and mortality by COVID-19 concern family provision and consequent occupational exposure at work, unhealthy lifestyle, expressed by smoking and alcoholism, violation of social distance rules, neglect of practices preventive measures, which reverberate in the weakened male immune system. However, the literature also revealed that the lack of male search for health services influenced by the conviction of invulnerability and by the political discourses that alleviate the disease are also influencing the increase in male exposure and mortality by COVID-19. The knowledge of the elements that favor men to illness and consequent death by the disease, urgent that they be thought of prevention for this public.

DESCRIPTORES: Infecciones por Coronavirus; Salud del Hombre; Indicadores de Morbimortalidad.

RECEBIDO EM: 29/01/2021 APROVADO EM: 09/02/2021 


\section{Andréia de Jesus Soares}

Enfermeira. Pós graduada em Unidade de Terapia intensiva e em Gerenciamento de Resíduos Sólidos em Saúde. Secretaria Municipal de Saúde de Salvador, Bahia.

ORCID: 0000-0002-9299-1678

\section{Caroline Fernandes Soares e Soares}

Graduanda em enfermagem pela Universidade Estadual de Feira de Santana.

ORCID: 0000-0003-4464-8389

\section{Fernanda Caetano dos Santos Silva}

Graduanda em enfermagem pela Universidade Estadual de Feira de Santana.

ORCID: 0000-0002-6074-8665

\section{Andrey Ferreira da Silva}

Enfermeiro. Pós-doutorando em Enfermagem pelo Programa de Pós-Graduação em Enfermagem da Universidade Federal da Bahia - PPGENF/UFBA. Professor da Universidade Federal de Alagoas, Arapiraca, AL, Brasil.

ORCID: 0000-0002-1038-7443

\section{Fernanda Matheus Estrela}

Enfermeira. Doutora em Enfermagem e Saúde. Docente da Universidade Estadual de Feira de Santana.

ORCID: 0000-0001-7501-6187

\section{Júlia Renata Fernandes de Magalhães}

Enfermeira. Doutora em Enfermagem. Universidade Federal da Bahia.

ORCID: 0000-0003-0631-2374

\section{Milena Arão da Silva Oliveira}

Enfermeira. Mestranda pela Universidade Federal da Bahia.

ORCID: 0000-0003-3933-991X

\section{Anderson Moreira Lima}

Enfermeiro da Estratégia Saúde da Família. Secretaria Municipal de Saúde do Salvador. ORCID: 0000-0002-0793-8881

\section{INTRODUÇÃO}

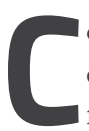
onfigurando-se como uma pandemia, que tem se propagado de forma rápida e devastadora, a COVID-19, causada pelo SARS-CoV-2, foi detectada em 31 de dezembro de 2019 em Wuhan, na China. Mediante a sua rápida velocidade de propagação por todo o mundo, foi declarado pela Organização Mundial da Saúde (OMS) uma Emergência de Saúde Pública de Importância Internacional $^{(1)}$, ocasionando, até o dia 01 de junho de 2020, mais de 6 milhões de casos e mais de 370 mil mortes distribuídos no mundo ${ }^{(2)}$. No Brasil, é importante considerar a característica ascensional e veloz da curva epidêmica, com mais de 391 mil casos confirmados e mais de 24 mil mortes, até o dia 26 de maio de $2020^{(3)}$, sendo, o público masculino, o mais afetado pela doença.

A morbimortalidade masculina por COVID-19 é superior às femininas em diversos países do mundo. Estudos revelam que na China, França, Alemanha, Iran, Itália e Coreia do Sul e Brasil cerca de $60 \%$ dos óbitos são do sexo masculino ${ }^{(4,5)}$. Em outras epidemias, onde as infecções se deram de forma mais tardia como no Oriente Médio, à mortalidade masculina mais elevada já havia sido detectada o que revela maior vulnerabilidade masculina ao desenvolvimento da doença ${ }^{(6)}$.

A vulnerabilidade em saúde reconhece que, diante das dimensões sociais, político-institucionais e comportamentais, diferentes pessoas e grupos populacionais apresentam suscetibilidades diferenciadas ainda que interligadas. A vulnerabilidade passa a se articular em três eixos: individual, social e programático(7). A dimensão individual engloba o conhecimento e os comportamentos acerca do agravo assumido pela pessoa, na qual lhe direciona ao evento de risco(7). A dimensão social relaciona a conduta individual com as características do espaço social, tais como: ciclo de vida, identidade social, normas sociais e institucionais, relações de gênero, entre outros aspectos ${ }^{(7)}$. E a dimensão pragmática refere-se ao acesso ao sistema de saúde, as ações preconizadas para a prevenção e o controle do agravo e os recursos sociais existentes na área de abrangência do serviço de saú$\mathrm{de}^{(7)}$. Ressalta-se que foram utilizados os conceitos de vulnerabilidade em sua tridimensionalidade

Esses dados podem guardar relação com os elementos que constituem o processo de formação da identidade de gê- 
nero masculina, visto que os homens são construídos socialmente para ocuparem os espaços públicos, locais estes que, no contexto da pandemia, são considerados lócus de transmissão.

Além disso, historicamente o cuidado de si não é uma prática comum dos homens que tendem a maior exposição aos fatores de risco à saúde, sendo a procura por assistência médica empreendida em uma fase de maior gravidade da doença ${ }^{(8)}$. Estudos reforçam tais achados quando revelam que nas Américas os homens vivem 5,8 anos a menos que as mulheres, considerando que estes têm comportamentos tóxicos à sua saúde ${ }^{(9)}$. Nesse sentido o olha para os aspectos da saúde sob a ótica do gênero enquanto categoria analítica se mostra importante quando considera as dimensões sociais, produtivas, políticas e culturais, reverberando nas práticas e saberes em saúde ${ }^{(8)}$

$\mathrm{Na}$ atual conjuntura, mesmo com as recomendações da OMS e do Ministério da Saúde Brasileiro para a quarentena e o distanciamento social, os homens são o público que mais se expõe. Pesquisa realizada pelo Instituto Datafolha revelam que as mulheres respeitam mais o distanciamento social do que os homens ${ }^{(10)}$. Isto implica dizer que estes resistem à adoção de práticas de autocuidado, negligenciando as medidas preventivas de higiene essenciais durante a pandemia, bem como ignorar sintomatologias brandas da doença e os alertas das autoridades de saúde. Dessa forma, tem-se como questão de pesquisa: Quais elementos da masculinidade que vulnerabilizam homens a morbimortalidade pela COVID-19?

Nesse sentido, o presente artigo tem por objetivo: Conhecer os elementos da masculinidade que vulnerabilizam homens a morbimortalidade pela $\mathrm{CO}$ VID-19.

\section{MÉTODOS}

Estudo de revisão integrativa da literatura que permite a síntese das evidências científicas acerca de determinada temática a partir da busca e análise ampliada de uma variedade de estudos. Pelo contexto de isolamento social, o que dificulta o acesso à homens para a realização de uma pesquisa empírica, a busca na literatura, por meio da revisão integrativa, permite a compilação de informações relevantes, favorecendo ao pesquisador a compreensão mais abrangente sobre a problemática para subsidiar à prática da atenção em saúde. Nesse sentido, metodologia se propõe ao apontamento de lacunas no conhecimento, dando ao pesquisador o direcionamento aos temas que necessitam de exploração científica ${ }^{(11)}$.

O estudo seguiu as seis etapas preconizadas a realização de uma revisão integrativa, sendo elas: Identificação do tema e seleção da questão de pesquisa; estabelecimento de critérios de inclusão e exclusão para seleção dos materiais científicos; categorização dos estudos; avaliação dos estudos incluídos; interpretação dos resultados e apresentação da síntese do conhecimento de acordo com o Revised Standards for Quality Improvement Reporting Excellence (SQUIRE 2.0) ${ }^{(12)}$.

Para a primeira etapa, utilizou-se a estratégia PICO que representa um acrônimo para Paciente (P), Intervenção (I), Comparação $(\mathrm{C})$ e "Outcomes" $(\mathrm{O})$ desfecho, sendo esses elementos fundamentais da questão de pesquisa e da construção da pergunta orientadora do estudo ${ }^{(13)}$. Desse modo, tendo como base tal

Quadro 1: Aplicação da estratégia PICO

\begin{tabular}{|c|c|c|}
\hline ACRÔNIMO & DEFINIÇÃo & APLICAÇÃo \\
\hline P & População & Homens \\
\hline I & Intervenção & COVID-19 \\
\hline C & Comparação & Não se aplica \\
\hline O & Desfecho & Morbimortalidade pela COVID-19 \\
\hline
\end{tabular}

estruturação, a pesquisa foi guiada pela seguinte questão: que elementos da masculinidade que vulnerabilizam homens a morbimortalidade pela COVID-19?. Salienta-se que não consideramos a vertente "C", visto que este estudo não se propõe ao desenvolvimento de pesquisas clínicas, o que torna dispensável sua utilização ${ }^{(14)}$.

O levantamento bibliográfico foi realizado no mês de maio de 2020, por meio da consulta na Plataforma Pubcovid-19, a qual está indexada na Biblioteca Nacional de Medicina dos Estados Unidos (PUBMED) e no Banco de Dados Excerptar Medica (EMBASE). Além disso, realizou-se a busca na Biblioteca Virtual em Saúde (BVS), a qual reúne as principais bases de dados que publicam sobre assuntos pertinentes à saúde a nível nacional e internacional, garantindo a variedade dos resultados que serão encontrados. Para se chegar às publicações sobre a temática, buscou-se selecionar estudos utilizando os Descritores em Ciências da Saúde (DECS): "Covid", "Gender Identity", "Men" e "Health", utilizando as seguintes estratégias de busca "Covid" And "Men"; "Covid" And "Men" And "Health"; "Covid" And "Gender Identity".

Constituíram-se os seguintes critérios de inclusão: artigos disponíveis na íntegra e de forma gratuita que versavam acerca da temática elementos da masculinidade que vulnerabilizam homens a morbimortalidade pela COVID-19, publicados no ano de 2020 e, apresentadas no idioma inglês. Assim, foram excluídos os artigos que não versavam sobre a temática $\mathrm{em}$ voga ou que não evidenciaram de forma clara os elementos pertinentes à pesquisa e literaturas cinzentas. A triagem dos resultados se deu por meio da leitura dos títulos e resumos, realizado por três autores de forma independente, adotando-se a revisão por pares. Por último, as avaliações foram avaliadas por meio da análise de conteúdo para o levantamento e síntese dos conhecimentos.

\section{RESULTADOS}

A análise dos dados perpassou pela 
síntese dos estudos selecionados e a realização de comparações dos principais resultados que respondiam à questão de pesquisa. Além disso, achados foram interpretados tomando como base o referencial teórico do gênero, com vistas às discussões das masculinidades, relacio- nando-as com o contexto da pandemia do COVID-19 e o descuidado masculino em relação a sua saúde.

Ao realizar a busca na BVS, foram obtidos 215 artigos. Ao efetivar a mesma busca na base de dados PubMed, foram obtidos 275 artigos, totalizando em 490

\section{Figura 1 - Fluxograma de identificação e seleção dos artigos para revisão integrativa, 2020.}

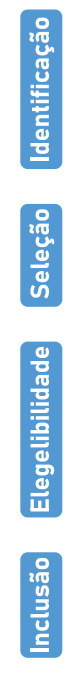

Covid AND Gender Identity Covid AND Men AND health Covid AND Men $\downarrow$

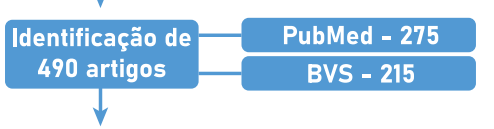

Duplicados- 11 $\downarrow$

Publicações não completas e gratuitas, fora do período, em outro idioma- 147

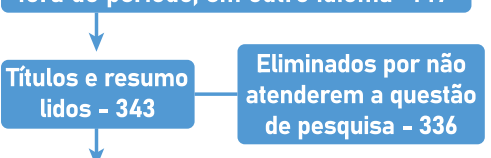

Artigos incluídos-7
Critérios de inclusão: publicações completas com tema relacionado aos elementos da masculinidade que vulnerabilizam homens a morbimortalidade pela COVID-19; publicadas no ano de 2020; disponiveis gratuitamente na integra; e apresentadas no idioma inglês.

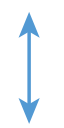

Critérios de Exclusão: artigos em que as repercussöes näo estivessem relacionadas ao objeto de estudo, além dos duplicados, teses, dissertaçőes, livros e/ou outras publicaçōes não originais

Fonte: Autoria própria, 2020.

documentos. Após a aplicação dos critérios de inclusão e exclusão previamente estabelecidos, restaram 343 documentos, dos quais, apenas 07 abordavam a temática central da pesquisa e fizeram parte da construção do estudo.

Destaca-se que a seleção foi guiada por um checklist elaborado pelos autores da pesquisa com informações relevantes dos artigos. O processo de identificação e seleção dos estudos seguiu as recomendações do Preferred Reporting Items for Systematic Reviews and Meta Analyses (PRISMA) ${ }^{(15)}$ conforme demonstra a figura 1 a seguir:

Com base nas sete publicações selecionadas, foi desenvolvido um quadro expondo às características dessas publicações segundo título, autoria, ano de publicação, país, periódico, metodologia adotada no estudo e os resultados encontrados dentre os artigos analisados.

\section{DISCUSSÃO}

De acordo com a literatura científica pesquisada, um dos elementos da masculinidade que incitam a vulnerabilidade do homem à morbimortalidade pela COVID-19 relaciona-se com o provi-

\begin{tabular}{|c|c|c|c|c|}
\hline $\mathbf{N}^{\circ}$ & TÍTULO & $\begin{array}{c}\text { AUTORES, ANO, REVISTA, } \\
\text { PAIIS }\end{array}$ & MÉTODO & RESULTADOS \\
\hline 1 & $\begin{array}{l}\text { Sex Differences in Mortality } \\
\text { from COVID-19 Pandemic: } \\
\text { Are Men Vulnerable and } \\
\text { Women Protected? }{ }^{(16)}\end{array}$ & $\begin{array}{c}\text { Sharma G, Volgman AS, } \\
\text { Michos ED. } 2020 \text { JACC Case } \\
\text { Rep, Estados Unidos }\end{array}$ & $\begin{array}{l}\text { Estudo de } \\
\text { caso }\end{array}$ & $\begin{array}{c}\text {-Doenças pré-existentes; } \\
\text {-Tabagismo e consumo excessivo de álcool; } \\
\text {-Provimento familiar e consequente exposição ocu- } \\
\text { pacional no trabalho; } \\
\text {-Negligência às medidas preventivas de higiene; } \\
\text {-Buscam menos os serviços de saúde. }\end{array}$ \\
\hline 2 & $\begin{array}{c}\text { Impact of sex and gender } \\
\text { on COVID-19 outcomes in } \\
\text { Europe } \text { e }^{(17)}\end{array}$ & $\begin{array}{l}\text { Gebhard C, Regitz-Zagrosek } \\
\text { V, Neuhauser HK, Morgan } \\
\text { R, Klein SL. } 2020 \text { Biol Sex } \\
\text { Differ, Inglaterra }\end{array}$ & $\begin{array}{l}\text { Revisão } \\
\text { narrativa }\end{array}$ & $\begin{array}{l}\text {-Tabagismo e consumo excessivo de álcool; } \\
\text {-Negligencia as medidas preventivas de higiene; } \\
\text {-Buscam menos os serviços de saúde; } \\
\text {-Rejeição ao distanciamento social, } \\
\text {-Provimento familiar e consequente exposição } \\
\text { ocupacional no trabalho; }\end{array}$ \\
\hline 3 & Men hit harder by covid-19(18) & $\begin{array}{c}\text { Lawton G. } 2020 \text { New Sci, } \\
\text { Inglaterra }\end{array}$ & $\begin{array}{l}\text { Revisão } \\
\text { Narrativa }\end{array}$ & $\begin{array}{c}\text {-Tabagismo; } \\
\text {-Doenças pré-existentes; -Negligenciam as medidas } \\
\text { preventivas de higiene } \\
\text {-Sistema imunológico frágil }\end{array}$ \\
\hline 4 & $\begin{array}{l}\text { Are we equal in adversity? } \\
\text { Does Covid-19 affect women } \\
\text { and men differently? }\end{array}$ & $\begin{array}{l}\text { Serge R, Vandromme J, } \\
\text { Charlotte M } 2020 \text { Irlanda }\end{array}$ & Reflexão & $\begin{array}{c}\text {-Sistema imunológico frágil } \\
\text {-Negligenciam as medidas preventivas de higiene } \\
\text {-Tabagismo } \\
\text {-Doenças pré-existentes }\end{array}$ \\
\hline
\end{tabular}




\begin{tabular}{|c|c|c|c|c|}
\hline 5 & $\begin{array}{c}\text { Men and COVID-19: Adding a } \\
\text { gender lens }{ }^{(20)}\end{array}$ & $\begin{array}{c}\text { Betron M, Gottert A, } \\
\text { Pulerwitz J, Shattuck D, Ste- } \\
\text { vanovic-Fenn N 2020 Glob } \\
\text { Public Health; Reino Unido }\end{array}$ & Reflexão & $\begin{array}{c}\text {-Tabagismo e consumo excessivo de álcool; } \\
\text {-Doenças pré-existentes; } \\
\text {-Negligência às medidas preventivas de higiene }\end{array}$ \\
\hline 6 & $\begin{array}{c}\text { COVID-19: the gendered } \\
\text { impacts of the outbreak }{ }^{(21)}\end{array}$ & $\begin{array}{c}\text { Wenham C, Smith J, Morgan } \\
\text { R. 2020 Lancet; Reino Unido }\end{array}$ & $\begin{array}{c}\text { Revisão } \\
\text { Narrativa }\end{array}$ & $\begin{array}{c}\text {-Sistema imunológico frágil; } \\
\text {-Tabagismo. }\end{array}$ \\
\hline 7 & $\begin{array}{c}\text { COVID-19 Worries and Beha- } \\
\text { vior Changes in Older and } \\
\text { Younger Men and Women (22) }\end{array}$ & $\begin{array}{c}\text { Barber SJ, Kim H 2020 J } \\
\text { Gerontol B Psychol Sci Soc } \\
\text { Sci; Estados Unidos }\end{array}$ & $\begin{array}{c}\text { Estudo } \\
\text { quantitativo }\end{array}$ & $\begin{array}{c}\text { Negligência às mudanças de comportamento para } \\
\text { cumprir as medidas preventivas } \\
\text {-Questões hormonais e sistema imunológico mais } \\
\text { frágil }\end{array}$ \\
\hline
\end{tabular}

mento familiar e sua consequente exposição às atividades laborais o que aumenta o risco ao contágio por COVID-19. Este contexto guarda relação com o fato dos homens serem construídos socialmente para ocupar espaços de âmbito público na tentativa de prover as demandas financeiras do $\operatorname{lar}^{(23)}$.

Tal perspectiva é apontada nos estudos nacionais e internacionais quando denotam que desde tenra idade os meninos são incentivados a ocuparem os espaços públicos por meio exposição a partir das brincadeiras de infância a símbolos subjetivos que instigam a liberdade de ir e vir bem como a importância do trabalho remunerado ${ }^{(16,17)}$. $\mathrm{Na}$ atual conjuntura, estar em espaços públicos para o desenvolvimento de qualquer atividade, seja de trabalho ou laser, expõe a população masculina ao adoecimento à COVID-19 o que denota a multideterminação da concepção de vulnerabilidade.

Para além das questões relacionadas ao labor, outro elemento apontado pela literatura como catalisador da morbimortalidade masculina pela COVID-19 diz respeito aos hábitos não saudáveis expressos pelo alcoolismo e tabagismo. A literatura internacional que versa sobre a COVID-19 desponta para a maior incidência dos homens que fumam ou bebem adoecerem $^{(16,17,18,19,20,21)}$. A convivência social com essas substâncias é milenar e cultural, estando associada a eventos sociais. Além disso, seu consumo remete a situações prazerosas, de alegria e de pertencimento a grupos sociais atrativos, facilitando o processo, principalmente masculino, de gregária com outros homens ${ }^{(24)}$. Diante do contexto da pande- mia, onde há necessidade do distanciamento social, os homens não conseguem se socializar em bares e/ou restaurantes, fazendo com que esses busquem outras estratégias para tal, optando, muitas vezes, pela a organização de festas privadas com várias pessoas.

Essa socialização, e consequente violação das regras de distanciamento social desvelam a negligência masculina as práticas preventivas preconizadas internacionalmente contra a COVID-19. Logo, a população masculina se mostra resistente ao cumprimento do distanciamento social, rejeitando não só a necessidade de se isolar, como também as orientações de higiene com a lavagem das mãos e o uso do álcool em gel, reforçando ainda mais o descuidado masculino com a saúde ${ }^{(16,17,19,22,25)}$. A identidade masculina sempre esteve associada ao descuidado com a aparência, vestimentas e higiene, visto sua conotação à virilidade e heterosexualidade, considerando tais cuidados estritamente feminino, todavia, na contemporaneidade, esses comportamentos têm mudado ${ }^{(26)}$. Apesar disso, muitos homens ainda associam a adoção de hábitos de higiene e cuidados a conotação de uma possível homosexualidade ${ }^{(27)}$. Esse contexto revela o eixo vulnerabilidade voltado ao campo social, uma vez que essa guarda relação com condutas individuais que repercutem de forma direta em todos os contextos social desse indivíduo. Nesse sentido, na atual conjuntura em que a adoção desses hábitos é ideal ao combate e controle da COVID-19, ao ignorar essas práticas de asseio, essa população está suscetível ao adoecimento.

Todas essas condutas negligentes, so- madas ao estilo de vida não saudável debilitam ainda mais o sistema imunológico masculino, favorecendo a progressão e os resultados adversos ao novo coronavírus, conforme desponta a literatura selecionada. Evidências sugerem diferenças substanciais no sistema imunológico entre os sexos, em que as mulheres desenvolverem defesas imunológicas mais fortes devido às questôes hormonais que desempenham um papel essencial na imunidade inata e adaptativa do organismo à uma gama de doenças infecciosas ${ }^{(18-21)}$. Logo, associado à uma alta exposição à patógenos, frequente adoecimento e baixa qualidade de vida, fica constatado que as condições de saúde preexistentes fundamentais para o agravamento dos sintomas da COVID-19 são mais prevalentes $\mathrm{e}$ incidentes no gênero masculino ${ }^{(16,17-20)}$.

A conotação da masculinidade à invulnerabilidade, construída socialmente e apreendida pelos homens é condição catalisadora ao descuidado dos homens com sua saúde, favorecendo ao não reconhecimento de situaçóes de alerta e a necessidade de buscar atendimento aos serviços de saúde. A dificuldade masculina em buscar assistência médica guarda relação, em sua maioria, ao fato do funcionamento dos serviços de saúde colidir com o horário de trabalho do homem ${ }^{(29)}$. Para além disso, procurar por assistência à saúde denota fragilidade, considerando que o papel social de masculinidade se pauta na força e invulnerabilidade, o que dificulta o reconhecimento de suas necessidades de cuidado à saúde ${ }^{(30)}$.

Para além das diferenças biológicas entre homens e mulheres, o discurso político de abreviação da gravidade dos sin- 
tomas, associando-a a uma "gripezinha", desponta na população, principalmente a masculina, o descrédito aos cuidados que devem ser tomados, bem como a letalidade da doença. À literatura retrata que frente à realidade, os líderes políticos possuem um papel fundamental na transmissão de informações à população em relação a doença. Quando estes não atuam conforme em concordância com a real conjuntura apresentada geram uma sensação de incompetência e falta de discernimento quanto à importância das ciências em detrimento às orientações dadas na tentativa de mitigar os efeitos econômicos em detrimento dos regimes da quarentena ${ }^{(31)}$.

Essa conjuntura faz com que os homens subestimam a gravidade do vírus considerando a sintomatologia branda presente inicialmente. Todavia, a COVID-19 apresenta evolução rápida levando qualquer indivíduo, seja homem ou mulher a quadros de gravidade dos sintomas, considerando uma série de circunstâncias clínicas individuais que interferem nesse processo, necessitando de cuidados intensivos, principalmente por complicações respiratórias ${ }^{(31)}$. Nesse ínterim, de acordo com as instâncias internacionais, ainda que seja considerado os fatores individuais, os homens são mais propensos a desenvolver a forma mais graves de COVID-19, necessitando de cuidados intensivos, sendo mais propensos a letalidade do vírus ${ }^{(19,33,34)}$.

Esse cenário revela a importância de reconhecer o impacto do COVID-19 na saúde dos homens, sendo de fundamental importância o fortalecimento de estratégias de prevenção e sensibilização desse público no tocante a gravidade dessa doença.

\section{CONCLUSÃO}

De acordo com a literatura científica explorada, os elementos da masculinidade que incitam a vulnerabilidade do homem à morbimortalidade pela COVID-19 dizem respeito ao provimento familiar e sua consequente exposição ocupacional no trabalho, estilo de vida não saudável, expressos pelo tabagismo e alcoolismo, violação das regras de distanciamento social, negligência de práticas preventivas à exemplo da lavagem das mãos, que reverberam no sistema imunológico masculino mais debilitado. Entretanto à literatura também desvelou que a não busca masculina pelos serviços de saúde influenciado pela convicção de invulnerabilidade e pelos discursos políticos que minoram a doença também são influenciadoras na elevação da exposição e mortalidade masculina pela COVID-19.

Uma das limitações do estudo refere-se à quantidade de artigos selecionados metodologicamente relacionando-os elementos da masculinidade que vulnerabilizam homens a morbimortalidade pela COVID-19, visto que a maioria dos estudos aborda questões epidemiológicas o que requer estudos sobre essa temática.

Com o conhecimento dos elementos que favorecem os homens ao adoecimento e consequente morte pela doença, urge que sejam pensadas estratégias de prevenção para esse público, desde o estímulo à mudança dos fatores de risco, incentivo à busca pelos serviços de saúde e orientações de medidas de higiene das mãos, uso de máscaras no trabalho para minimizar a transmissão do vírus para esse público. -

\section{REFERÊNCIAS}

1. Organização das Nações Unidas. OMS declara coronavírus emergência de saúde pública internacional [Internet]. Nações Unidas Brasil: 2020 [citado 2020 jun 09]. Disponível em: https://nacoesunidas.org/oms-declara-coronavirus-emergencia-de-saude-publica-internacional/

2. Universidade Johns Hopkins. COVID-19 Dashboard by the Center for Systems Science and Engineering (CSSE) at Johns Hopkins University (JHU) [Internet]. Baltimore, Estados Unidos: Universidade Johns Hopkins, 2020 [citado 2020 jun 01]. Disponível em: https://coronavirus.jhu.edu/map.html

3. Brasil. Painel Coronavírus [Internet]. Ministério da Saúde, 2020 [citado 2020 mai 26]. Disponível em: https://covid.saude. gov.brl

4. Brasil. Ministério da Saúde. Boletim Epidemiológico n. 11 [Internet]. Ministério da Saúde, 2020 [citado 2020 jun 01]. Disponível em: https://www.saude.gov.br/images/pdf/2020/ April/18/2020-04-17---BE11---Boletim-do-COE-21h.pdf

5. Polglase K, Mezzofiore G, Foster M. Here's why the coronavirus may be killing more men than women. The US should take note [Internet]. Cable News Network, 2020 [cited 2020 jun 09]. Available from: https://edition.cnn.com/2020/03/24/health/ coronavirus-gender-mortality-intl/index.html

6. Brandão A. Ciência não sabe explicar por que coronavírus mata mais homens do que mulheres [Internet]. Rádio francesa de notícias, 2020 [citado 2020 jun 09]. Disponível em:http://www.rfi.fr/ br/fran\%C3\%A7a/20200420-ci\%C3\%AAncia-n\%C3\%A3o-sabeexplicar-por-que-coronav\%C3\%ADrus-mata-mais-homens-doque-mulheres

7. Ayres JRCM, Calazans GJ, Saletti Filho HC, França Júnior I. Risco, vulnerabilidade e práticas de prevenção e promoção da saúde. In: Campos GWS, Bonfim JRA, Minayo MCS, Akerman M, Drumond Júnior M, Carvalho YM, organizadores. Tratado de Saúde Coletiva. Rio de Janeiro: Fiocruz; 2006. p. 375-417.

8. Lemos AP, Ribeiro C, Fernandes J, Bernades K, Fernandes R. Saúde do homem: os motivos da procura dos homens pelos serviços de saúde. Rev enferm UFPE on line., 2017; 11(Supl. 11): 4546-53.

9. Organização Panamericana de Saúde. Masculinidades y salud en la Región de las Américas. Resumen. Washington, 2019 [citado 2020 jun 06].Disponivel em: https://iris.paho.org/handle/10665.2/51667 


\section{artigo}

Soares, A.J.; Soares, C.F.S.; Santos Silva, F.C.; Ferreira da Silva, A.; Estrela, F.M.; Magalhães, J.R.F.; Oliveira, M.A.S.; Lima, A.M.

Elementos da masculinidade que vulnerabilizam homens à morbimortalidade pela COVID-19: revisão integrativa

\section{REFERÊNCIAS}

10. Almeida G. Coronavírus no Brasil: Mulheres respeitam mais o isolamento social, mostra pesquisa Datafolha [Internet] 0 povo Online, 2020 [citado 2020 jun 09]. Disponivel em: https://www. opovo.com.br/coronavirus/2020/04/28/coronavirus-no-brasil--mulheres-respeitam-mais-o-isolamento-social--mostra-pesquisa.html

11. Mendes KDS, Silveira RC de CP, Galvão CM. Use of the bibliographic reference manager in the selection of primary studies in integrative reviews. Texto Context - Enferm [Internet]. 2019 [cited 2020 Apr 16]; 28:e20170204. Available from: http://www.scielo.br/scielo.php?script=sci_arttext\&pid=S0104-07072019000100602\&tIng=en

12. Ogrinc G, Davies L, Goodman D, Batalden P, Davidoff F, Stevens D. SQUIRE 2.0 (Standards for QUality Improvement Reporting Excellence): revised publication guidelines from a detailed consensus process: Table 1. BMJ Qual Saf [Internet]. 2016 [cited 2020 jun 09]; 25(12): 986-92. Available from: http://qualitysafety.bmj.com/lookup/doi/10.1136/bmjqs-2015-004411

13. Santos CM da C, Pimenta CA de M, Nobre MRC. The PICO strategy for the research question construction and evidence search. Rev Lat Am Enfermagem [Internet]. 2007 Jun [cited 2018 May 15]; 15(3): 508-11. Available from: http://www.scielo.br/scielo. php?script=sci_arttext\&pid=S0104-11692007000300023\&Ing=en\&tlng=en

14. Greenhalgh T. Como ler artigos científicos: fundamentos da medicina baseada em evidências. 2a Ed. Porto Alegre: Artmed; 2005.

15. Galvão TF, Pansani T de SA, Harrad D. Principais itens para relatar Revisões sistemáticas e Meta-análises: A recomendação PRISMA. Epidemiol e Serviços Saúde [Internet]. 2015 [cited 2020 jun 09]; 24(2): 335-42. Available from: http://www. iec.pa.gov.br/template_doi_ess.php?doi=10.5123/S167949742015000200017\&scielo=S2237-96222015000200335

16. Sharma G, Volgman AS, Michos ED. Sex Differences in Mortality from COVID-19 Pandemic: Are Men Vulnerable and Women Protected? JACC Case Reports [Internet]. 2020. DOI: 10.1016/j. jaccas.2020.04.027

17. Gebhard C, Regitz-Zagrosek V, Neuhauser HK, Morgan R, Klein SL. Impact of sex and gender on COVID-19.outcomes in Europe. Biol Sex Differ. 2020; 11(1): 29. DOI: 10.1186/s13293020-00304-9

18. Lawton G. Men hit harder by covid-19. New Sci. 2020; 246(3279): 8. DOI: 10.1016/S0262-4079(20)30786-7

19. Serge R, Vandromme J, Charlotte M. Are we equal in adversity? Does Covid-19 affect women and men differently? Maturitas. 2020. DOI: 10.1016/j.maturitas.2020.05.009

20. Betron M, Gottert A, Pulerwitz J, Shattuck D, Stevanovic-Fenn N. Men and COVID-19: Adding a gender lens. Glob Public Health. 2020; 1-3. DOI: 10.1080/17441692.2020.1769702

21. Wenham C, Smith J, Morgan R. COVID-19: the gendered impacts of the outbreak. Lancet. 2020; 395(10227): 846-8. DOI: $10.1016 /$ S0140-6736(20)30526-2
22. Barber SJ, Kim H. COVID-19 Worries and Behavior Changes in Older and Younger Men and Women. Isaacowitz D, editor. Journals Gerontol Ser B. 2020. DOI: 10.1093/geronb/gbaa068

23. BrumleyKM. "It'sMoreAppropriatefor Men":Managementand Worker Perceptions of the Gendered Ideal Worker. Sociol Spectr. 2018; 38(6): 406-21. DOI: 10.1080/02732173.2018.1564096

24. Ribeiro KCS, Pereira LB, Wiese I, Silva J, Saldanha AAW. Consumo de álcool e tabaco e associação com outras vulnerabilidades em jovens. Psicol Saúde Doença. 2017; 18(2): 348-59. DOI: 10.15309/17psd180206

25. Paiva V. Por que o coronavírus está matando mais homens que mulheres [Internet]. hypeness, 2020 [citado 2020 jun 09]. Disponivel em: https://www.hypeness.com.br/2020/04/por-queo-coronavirus-esta-matando-mais-homens-que-mulheres/

26. Casanova EM de, Wetzel EE, Speice TD. Looking at the label: White-collar men and the meanings of "metrosexual." Sexualities. 2016; 19(1-2): 64-82. DOI: 10.1177/1363460715583607

27. Rosenmann A, Kaplan D, Gaunt R, Pinho M, Guy M. Consumer masculinity ideology: Conceptualization and initial findings on men's emerging body concerns. Psychol Men Masc. 2018; 19(2): 257-72. DOI: 10.1037/men0000095

28. Zhou Z, Chen P, Peng $H$. Are healthy smokers really healthy? Tob Induc Dis. 2016; 14(1): 35. DOI: 10.1186/s12971-0160101-z

29. Shwe Sin Ei W Le, Lwin Tun T, Htun C, Gignoux E, Thu Swe $K$, Incerti $A$, et al. Nagaland health assessment: High mortality rates and difficulty accessing essential health services in Lahe Township, Republic of the Union of Myanmar. Musinguzi G, editor. PLoS One. 2019; 14(5): e0216925. DOl: 10.1371/journal. pone.0216925

30. Lopes GSSP, Sardagna MC, lervolino SA. Motivos que levam os homens a procurar um serviço de pronto atendimento. Enfermagem Revista [Internet]. 2017 [citado 2020 jun 09]; 20(2). Disponivel em: http://periodicos.pucminas.br/index.php/enfermagemrevista/article/view/16331

31. Troi M, Quintilio W. Coronavírus: lições anti-negacionistas e o futuro do planeta. [Internet]. SciELO em Perspectiva, 2020 [Citado 2020 jun 08]. Available from: https://blog.scielo. org/blog/2020/03/31/coronavirus-licoes-anti-negacionistas-e-o-futuro-do-planeta/

32. YouGov. YouGov Survey Results [Internet]. 2020 [cited 2020 jun 09]. Disponivel em: https://docs.cdn.yougov.com/tdmiiw5igp/Results $\% 20$ for $\% 20$ Internal\%20\%28Coronavirus $\% 20$ tracker\%29\%20w.pdf

33. Uk Research and Innovation. Sex, gender and COVID-19, 2020 [cited 2020 jun 09]. Available from: https://coronavirusexplained.ukri.org/en/article/cad0007/

34. Jin J-M, Bai P, He W, Wu F, Liu X-F, Han D-M, et al. Gender Differences in Patients With COVID-19: Focus on Severity and Mortality. Front Public Heal. 2020; 8. DOI: 10.3389/ fpubh.2020.00152 